\title{
A influência da Prática de Diferentes Atividades Físicas Sobre a Qualidade de Vida de Idosos
}

\author{
The Influence of the Practice of Different Physical Activities on the \\ Quality of Life of Elderly
}

\author{
José Antonio Bicca Ribeiro ${ }^{1}$ \\ Fernanda de Souza Teixeira ${ }^{2}$ \\ Adriana Schuler Cavalli ${ }^{3}$ \\ Mariângela da Rosa Afonso ${ }^{4}$
}

\section{RESUMO}

Objetivo: Verificar a influência da prática de atividade física em diferentes modalidades sobre a qualidade de vida de idosos. Metodologia: Participaram do estudo 132 idosos (72 $\pm 5,3$ anos; 117 mulheres) praticantes de atividade física orientada, realizada de forma contínua por pelo menos um ano nas modalidades: ginástica, hidroginástica e musculação. Todas as participantes do estudo responderam a um questionário sociodemográfico e aos instrumentos de qualidade de vida WHOQOL-Bref e WHOQOL-Old. Na comparação da qualidade de vida de acordo com as modalidades praticadas foi utilizado o teste de Kruskal Wallis, com nível de significância de $5 \%$. Resultados: O domínio com maior média de qualidade de vida foi o das relações sociais $(86,4 \pm 25,9)$, seguido pelo domínio das atividades passadas, presentes e futuras $(81,1 \pm 23,5)$. O domínio com menor média foi a morte e morrer $(50,4 \pm 35,7)$ e o psicológico $(75,7 \pm 20,8)$, além disso, a qualidade de vida total da amostra foi de $(67,4 \pm 12,6)$ pontos de média. Ocorreu uma associação da modalidade com a percepção da qualidade de vida, nos domínios do meio ambiente, funcionamento do sensório, morte e morrer, além da qualidade de vida total $(p<0,01)$. Nesse sentido as modalidades de ginástica e musculação apresentaram os melhores resultados em comparação com a hidroginástica. Conclusão: O tipo de atividade física realizada parece ter maior influência sobre os níveis de qualidade de vida da população idosa, mais do que o tempo de permanência nos programas.

\section{DESCRITORES}

Atividade Motora. Idosos. Estilo de Vida. Saúde.

\begin{abstract}
Objective: To verify the influence of physical activity in different modalities on the quality of life of the elderly. Methodology: The study included 132 elderly people ( $72 \pm 5.3$ years; 117 women) practicing guided physical activity, performed continuously for at least one year in the following modalities: gymnastics, water aerobics and weight training. All of them answered a sociodemographic questionnaire and the WHOQOL-Bref and WHOQOL-Old quality of life instruments. In the comparison of quality of life according to the modalities practiced, the Kruskal Wallis test was used with a significance of $5 \%$. Results: The domain with the highest average quality of life was Social Relations $(86.4 \pm 25.9)$, followed by the domain of Past, Present and Future Activities $(81.1 \pm 23.5)$. The domain with the lowest average was Death and Dying $(50.4 \pm 35.7)$ and Psychological (75.7 \pm 20.8$)$. In addition, the total quality of life of the sample was $67.4( \pm 12.6)$ mean points. There was an association between the modality and the perception of quality of life, in the domains of the Environment, Functioning of the Sensory, Death and Dying, in addition to the total quality of life $(p<0.01)$. In this sense, the modalities of gymnastics and bodybuilding showed the best results compared to water aerobics. Conclusions: More than the length of stay in the programs, the type of physical activity performed seems to have a greater influence on the quality-of-life levels of the elderly population.
\end{abstract}

\section{DESCRIPTORS}

Motor Activity. Elderly. Life Style. Health.

${ }^{1}$ Mestre em Educação Física. Doutorando do Programa de Pós-Graduação em Educação Física da Universidade Federal de Pelotas, Pelotas, RS, Brasil.

${ }^{2}$ Doutora em Ciências da Atividade Física e do Deporto pela Universidade de León, Espanha. Docente Associada da Escola Superior de Educação Física da Universidade Federal de Pelotas, Departamento de Ginástica e Saúde, Pelotas, RS, Brasil.

${ }^{3}$ Doutora em Ciências da Saúde e Esporte pela Chukyo University, Japão. Docente Associada da Escola Superior de Educação Física da Universidade Federal de Pelotas, Departamento de Desportos, Pelotas, RS, Brasil.

${ }^{4}$ Doutora em Educação pela Universidade Federal do Rio Grande do Sul. Docente Titular da Escola Superior de Educação Física da Universidade Federal de Pelotas, Departamento de Ginástica e Saúde, Pelotas, RS, Brasil. 
$\mathrm{O}$ envelhecimento é permeado por diversas mudanças de ordem física e psicológica como, por exemplo, a perda de massa magra aliado ao aumento do percentual de gordura, diminuição na capacidade funcional, aumento do sedentarismo, aumento do risco para o desenvolvimento de doenças crônico-degenerativas, além da diminuição da autoestima, interferindo no modo de vida da população idosa ${ }^{1-3}$. Atrelado a isso, percebe-se que existe um aumento na busca por alternativas que possam promover um envelhecimento mais saudável e a prática de atividade física têm demonstrado auxiliar nesse processo ${ }^{4-6}$.

Dentre os principais benefícios acarretados pela prática de AF regular podemos citar a recuperação de níveis de força muscular necessários para a realização das atividades de vida diária, diminuição nos riscos de quedas e consequentes fraturas, diminuição das taxas de morbidade e mortalidade, diminuição do risco para o desenvolvimento de doenças crônico-degenerativas, o resgate do sentimento de autoeficácia e autoestima, entre outros ${ }^{7-11}$. Fatores estes que repercutem positivamente na percepção que os indivíduos idosos têm da sua qualidade de vida ${ }^{11-13}$, entendida como "a percepção de bem-estar que reflete um conjunto de parâmetros individuais, socioculturais e ambientais que podem caracterizar as condições em que vive o ser humano"14.

Segundo Svantesson ${ }^{4}$, uma maior independência melhora a percepção de qualidade de vida e, melhores níveis de força muscular e de equilíbrio, beneficiam a independência. Estudo recente ${ }^{15}$ comparando idosos frágeis e robustos corroboram a afirmativa anterior. Entretanto, cabe destacar que não existe uma associação direta entre a melhora em capacidades físicas e a melhora no nível de qualidade de vida, o que reforça a ideia de que algumas dimensões da qualidade de vida podem ser mais ou menos estimuladas em função do tipo de atividade física pratica$\mathrm{da}^{16}$. Em contrapartida, parece que a maior aderência à prática de atividade física melhor será a qualidade de vida ao longo dos anos ${ }^{17}$. Portanto, o presente estudo teve por objetivo verificar a influência da prática de atividade física em diferentes modalidades sobre a qualidade de vida de idosos.

\section{METODOLOGIA}

O presente estudo, aprovado pelo Comitê de Ética em Pesquisa da Escola Superior de Educação Física sob o protocolo 047/2012, possui um caráter descritivo-exploratório do tipo transversal e foi realizado com idosos do Núcleo de Atividades para a Terceira Idade (NATI) da Escola Superior de Educação Física (ESEF/UFPel), na Cidade de Pelotas/RS. O NATI conta com aproximadamente 308 idosos em suas diferentes atividades, oferecidas nas modalidades: ginástica, hidroginástica e musculação.

Todos os integrantes do NATI foram convidados a participar da pesquisa e foram considerados como critérios de inclusão: ter 60 anos de idade ou mais, assinar o Termo de Consentimento Livre e Esclarecido (TCLE) e participar de todas as etapas da pesquisa. Atendendo a estes critérios a amostra foi composta por 132 idosos (117 mulheres), com média de idade de $72 \pm 5,3$ anos, divididos nas modalidades: ginástica $(n=31)$, hidroginástica $(n=85)$ e musculação $(n=16)$. 
Para a coleta de dados, foram utilizados três instrumentos: um questionário com informações sociodemográficas e de adesão ao programa (sexo, idade, situação conjugal, tempo de permanência no programa, percepção de saúde autorreferida, comorbidades e consumo de medicamentos; questionário de qualidade de vida WHOQOL-Old; questionário de qualidade de vida WHOQOL-Bref.

O WHOQOL-Old ${ }^{18}$ é composto por 24 questões compreendendo seis domínios (funcionamento do sensório; autonomia; atividades passadas, presentes e futuras; participação social; morte e morrer e intimidade) além de uma média geral da qualidade de vida. Para cada questão existe uma escala do tipo likert que atribui os valores de 1 a 5 às respostas, sendo 1 (um) a pior representação da qualidade de vida em cada domínio e 5 (cinco) a melhor. $O$ cálculo dos escores de cada domínio, bem como da avaliação geral foi realizado, de acordo com o manual de aplicação dos instrumentos ${ }^{18}$, adotando a escala de exposição de 100 pontos.

O WHOQOL-Bref ${ }^{18}$ é composto por 24 questões compreendendo seis domínios (físico, psicológico, relações sociais e meio ambiente) e mais duas relacionadas a aspectos mais gerais da qualidade de vida. Para cada questão existe uma escala do tipo likert que atribui os valores de 1 a 5 às respostas, sendo 1 (um) a pior representação da qualidade de vida em cada domínio e 5 (cinco) a melhor. O cálculo dos escores de cada domínio, bem como da avaliação geral foi realizado, de acordo com o manual de aplicação dos instrumentos ${ }^{19}$, sendo adotado como valor máximo (melhor) o total de 100 pontos.

Cada participante respondeu aos ins- trumentos por meio de entrevista individual, no local onde realizava suas atividades e as entrevistas foram realizadas por entrevistadores previamente treinados.

Os dados coletados foram digitados em um banco no Microsoft Excel e transferidos para o STATA 12.1, onde foram realizadas as análises estatísticas. A estatística descritiva (média, mediana, desvio padrão, intervalos de confiança, frequência absoluta e relativa) foi utilizada para a exposição dos resultados referentes ao perfil sociodemográfico da amostra e aos domínios da qualidade de vida. Os dados tiveram distribuição não-paramétrica a partir do teste de Shapiro-Wilk. Na comparação dos domínios da qualidade de vida, de acordo com as modalidades, foi utilizado o teste de Kruska-Wallis. Para todas as análises foi adotado o nível de significância de 5\%.

\section{RESULTADOS}

Participaram do estudo 132 idosos (117 mulheres), com média de idade de $72 \pm 5,3$ anos, participantes do Núcleo de Atividades para a Terceira Idade, nas modalidades de ginástica $(n=31)$, hidroginástica $(n=85)$ e musculação $(n=16)$. Quanto às características sociodemográficas gerais da amostra, verificou-se que além da alta prevalência de indivíduos do sexo feminino (88,6\%), 50\% tem idade entre $60-70$ anos, $54,5 \%$ não tem companheiro (a), 78,0\% estão no projeto em um período de até dez anos.

Nas modalidades de ginástica e hidroginástica existe uma maior prevalência de mulheres (100\% e $92,9 \%$, respectivamente), enquanto que na musculação a maior prevalência é de homens (56,3\%). No que diz respeito à idade, nas modalidades de ginás- 
tica e musculação existe um maior percentual de sujeitos na faixa de $60-70$ anos $(74,2 \%$ e $81,3 \%$, respectivamente), enquanto na hidroginástica a maior prevalência é de sujeitos com 71 anos ou mais $(64,7 \%)$. No que diz respeito à situação conjugal, os participantes sem companheiro são mais prevalentes nas modalidades de ginástica $(58,1 \%)$ e hidroginástica $(56,5 \%)$, uma vez que na musculação a maior prevalência é de indivíduos com com- panheiro (62,5\%). Com relação ao tempo de adesão ao programa, a totalidade de sujeitos da ginástica e musculação, tem um tempo de adesão de até dez anos. No tocante à hidroginástica, existe um percentual menor de sujeitos com um tempo de participação superior a onze anos (34,1\%)

A presença de doenças foi identificada de acordo com atestado médico fornecido pelos participantes da pesquisa, no momen-

Tabela 1. Características sociodemográficas da amostra

\begin{tabular}{|c|c|c|c|c|c|c|c|c|}
\hline \multirow{3}{*}{ Variável } & \multicolumn{2}{|c|}{$\begin{array}{c}\text { Ginástica } \\
(n=31)\end{array}$} & \multicolumn{2}{|c|}{$\begin{array}{l}\text { Hidroginástica } \\
\quad(n=85)\end{array}$} & \multicolumn{2}{|c|}{$\begin{array}{c}\text { Musculação } \\
(n=16)\end{array}$} & \multicolumn{2}{|c|}{$\begin{array}{c}\text { Total } \\
(n=132)\end{array}$} \\
\hline & $\mathrm{n}$ & $\%$ & $\mathrm{n}$ & $\%$ & $\mathrm{n}$ & $\%$ & $\mathrm{n}$ & $\%$ \\
\hline & \multicolumn{8}{|c|}{$n=132$} \\
\hline \multicolumn{9}{|l|}{ Sexo } \\
\hline Masculino & - & - & 6 & 7,1 & 9 & 56,3 & 15 & 11,4 \\
\hline Feminino & 31 & 100 & 79 & 92,9 & 7 & 43,8 & 117 & 88,6 \\
\hline \multicolumn{9}{|l|}{ Idade } \\
\hline $60-70$ anos & 23 & 74,2 & 30 & 35,3 & 13 & 81,3 & 66 & 50,0 \\
\hline 71 anos ou mais & 8 & 25,8 & 55 & 64,7 & 3 & 18,8 & 66 & 50,0 \\
\hline \multicolumn{9}{|l|}{ Situação conjugal } \\
\hline Sem companheiro (a) & 18 & 58,1 & 48 & 56,5 & 6 & 37,5 & 72 & 54,5 \\
\hline Com companheiro (a) & 13 & 41,9 & 37 & 43,5 & 10 & 62,5 & 60 & 45,5 \\
\hline \multicolumn{9}{|c|}{ Tempo de adesão ao programa } \\
\hline Até dez anos & 31 & 100,0 & 56 & 65,9 & 16 & 100,0 & 103 & 78,0 \\
\hline Onze anos ou mais & - & - & 29 & 34,1 & - & - & 29 & 22,0 \\
\hline \multicolumn{9}{|l|}{ Comorbidades } \\
\hline Nenhuma doença & 1 & 3,2 & 4 & 4,7 & 2 & 12,5 & 7 & 5,3 \\
\hline Uma doença & 10 & 32,3 & 11 & 12,9 & 3 & 18,8 & 24 & 18,2 \\
\hline Duas doenças ou mais & 20 & 64,5 & 70 & 82,4 & 11 & 68,8 & 101 & 76,5 \\
\hline \multicolumn{9}{|l|}{ Quantidade de medicamentos } \\
\hline Nenhum & 2 & 6,5 & 5 & 5,9 & 3 & 18,8 & 10 & 7,6 \\
\hline Um medicamento & 5 & 16,1 & 10 & 11,8 & 3 & 18,8 & 18 & 13,6 \\
\hline Dois ou mais & 24 & 77,4 & 70 & 82,4 & 10 & 62,5 & 104 & 78,8 \\
\hline \multicolumn{9}{|l|}{ Percepção de saúde } \\
\hline Positiva & 26 & 83,9 & 58 & 68,2 & 13 & 81,3 & 97 & 73,5 \\
\hline Negativa & 5 & 16,1 & 27 & 31,8 & 3 & 18,8 & 35 & 26,5 \\
\hline
\end{tabular}


Tabela 2. Descrição dos escores de qualidade de vida através do WHOQOL-Bref e WHOQOL-Old

\begin{tabular}{|c|c|c|c|}
\hline Domínio & Média ( $\pm \mathrm{DP})$ & Med & IC (95\%) \\
\hline \multicolumn{4}{|l|}{ WHOQOL-Bref } \\
\hline \multirow{4}{*}{$\begin{array}{l}\text { Físico } \\
\text { Psicológico } \\
\text { Relações Sociais } \\
\text { Meio Ambiente }\end{array}$} & $75,6( \pm 21,5)$ & 71,4 & $71,9-79,3$ \\
\hline & $75,7( \pm 20,8)$ & 70,8 & $72,1-79,3$ \\
\hline & $86,4( \pm 25,9)$ & 83,3 & $82,0-90,9$ \\
\hline & $76,5( \pm 19,6)$ & 71,9 & $73,1-79,8$ \\
\hline \multicolumn{4}{|l|}{ WHOQOL-OId } \\
\hline \multirow{4}{*}{$\begin{array}{l}\text { Funcionamento do Sensório } \\
\text { Autonomia } \\
\text { Atividades passadas, presentes e futuras } \\
\text { Participação Social }\end{array}$} & $59,5( \pm 31,2)$ & 62,0 & $54,1-64,9$ \\
\hline & $73,4( \pm 18,8)$ & 75,0 & $70,1-76,6$ \\
\hline & $81,1( \pm 23,5)$ & 80,6 & $77,1-85,2$ \\
\hline & $74,9( \pm 16,9)$ & 75,0 & $72,0-77,9$ \\
\hline \multirow{2}{*}{$\begin{array}{l}\text { Morte e Morrer } \\
\text { Intimidade }\end{array}$} & $50,4( \pm 35,7)$ & 53,1 & $44,2-56,5$ \\
\hline & $74,3( \pm 23,8)$ & 75,0 & $70,2-78,4$ \\
\hline QV Total & $67,4( \pm 12,6)$ & 66,7 & $65,2-69,5$ \\
\hline
\end{tabular}

Med=mediana; IC=intervalo de confiança.

to da coleta de dados. Apenas sete sujeitos indicaram não ter nenhuma enfermidade, considerando toda a amostra. A maior prevalência nas modalidades de ginástica $(64,5 \%)$, hidroginástica $(82,4 \%)$ e musculação $(68,8 \%)$ foi de indivíduos com duas ou mais comorbidades. Sobre as comorbidades, a amostra possui uma prevalência de $64,4 \%$ de hipertensão arterial sistêmica, sendo esta, a principal enfermidade enfrentada pelos sujeitos.

Cabe ressaltar que os demais tipos de doenças mais prevalentes foram doenças osteomusculares $(48,5 \%)$ seguidas pelas doenças metabólicas $(41,7 \%)$. Outros tipos de quadros clínicos como cardiopatias, disfunções no aparelho vestibular, enfermidades no sistema respiratório/digestivo, problemas de audição/visão, doenças psicossomáticas, assim como, alguns casos de metástase, tiveram prevalências inferiores às demais enfermidades.

Associado às enfermidades está o uso de medicamentos e, apenas, dez sujeitos $(7,6 \%)$ não fazem uso de medicamento no seu cotidiano. Quando estratificamos de acordo com a modalidade ginástica, hidroginástica e musculação, a maior prevalência em todas $(77,4 \%, 82,4 \%$ e $62,5 \%$, respectivamente) foi de indivíduos que fazem uso de dois medicamentos ou mais.

A percepção de saúde da amostra foi obtida de forma autorreferida e agrupamos a mesma segundo aspectos negativos (péssima, ruim e regular) e aspectos positivos (boa e ótima). Entre todos os sujeitos participantes a percepção de saúde foi positiva, sendo que o maior percentual foi entre os praticantes de ginástica $(83,9 \%)$, seguidos pelos praticantes da musculação $(81,3 \%)$ e hidroginástica $(68,2 \%)$.

Considerando a avaliação da qualidade de vida por meio do WHOQOL-Bref e WHOQOL-Old, apresentamos a Tabela 2, com os valores de média e desvio padrão 
para cada domínio, bem como, os valores de mediana e intervalos de confiança. Entre os domínios do WHOQOL-Bref, o que apresentou maior média, entre todos, foi o das relações sociais $[86,4( \pm 25,9) ; \mathrm{IC}=(82,0-90,9)]$, sendo este domínio caracterizado pela percepção que os indivíduos tem da capacidade de se relacionar e se envolver com outras pessoas. Por outro lado, o domino com menor média de qualidade de vida foi o físico $[75,6( \pm 21,5)$; IC=(71,9 - 79,3)], responsável pela percepção que os sujeitos tem sobre o que sentem com relação ao seu corpo e a capacidade de executar tarefas cotidianas.

A Tabela 3 traz os resultados referentes à comparação entre os domínios de qualidade de vida do WHOQOL-Bref, de acordo com a modalidade praticada. Entre todos os domínios, apenas o domínio do meio ambiente apresentou diferença estatisticamente significativa $(p<0,01)$, quando comparado às modalidades de ginástica, hidroginástica e musculação. Neste domínio, a maior média de qualidade de vida foi entre os praticantes de hidroginástica $(80,6 \pm 22,0$; Med $=75,0)$, seguidos pelos praticantes da ginástica $(69,8 \pm 11,4$; Med=68,7) e musculação $(67,8 \pm 10,0 ;$ Med=68,7).
Considerando todas as modalidades, o domínio que apresentou as melhores médias foi o das relações sociais. $\mathrm{Na}$ modalidade ginástica a média foi de 80,1 pontos ( $D P=15,5$; Med=83,3); na modalidade hidroginástica a média foi de 89,15 pontos $(\mathrm{DP}=30,2 ; \mathrm{Med}=83,3)$; e na modalidade de musculação a média foi de 54,4 pontos (DP=11,3; Med=87,5). O domínio com menor média na modalidade de hidroginástica foi o físico $(73,04 \pm 12,6$; Med=71,4), sendo que nas demais modalidades, as menores médias foram encontradas no domínio do meio ambiente, tanto na ginástica $(69,8 \pm 11,4$; Med=68,7) quanto na musculação $(67,8 \pm 10,0$; Med=68,7).

A Tabela 4 apresenta os resultados referentes aos domínios do WHOQOL-Old de acordo com a modalidade de atividade física praticada pelos participantes da pesquisa. Ocorreram diferenças estatísticas nos domínios do funcionamento do sensório, morte e morrer e na avaliação da qualidade de vida total fornecida pelo instrumento $(p<0,01)$.

Considerando o domínio do funcionamento do sensório a maior média pode ser encontrada entre os praticantes de musculação $(82,8 \pm 18,9$; Med=90,6), seguido pela média

Tabela 3. Descrição dos domínios da qualidade de vida do WHOQOL-Bref de acordo com a modalidade de atividade física praticada

\begin{tabular}{l|c|c|c|c|c|c|c}
\hline \multirow{2}{*}{$\begin{array}{c}\text { Domínios do } \\
\text { WHOQOL-Bref }\end{array}$} & \multicolumn{2}{|c|}{ Ginástica } & \multicolumn{2}{c|}{ Hidroginástica } & \multicolumn{2}{c|}{ Musculação } & \multirow{2}{*}{ * } \\
\cline { 2 - 8 } & Média $( \pm D P)$ & Med & Média $( \pm D P)$ & Med & Média $( \pm D P)$ & Med & \\
\cline { 2 - 8 } & $73,0( \pm 12,6)$ & 71,4 & $76,3( \pm 24,8)$ & 67,9 & $77,0( \pm 15,7)$ & 78,6 & 0,253 \\
\cline { 2 - 8 } Físico & $72,0( \pm 10,6)$ & 70,8 & $77,0( \pm 24,2)$ & 70,8 & $76,0( \pm 14,8)$ & 79,2 & 0,664 \\
\cline { 2 - 7 } Psicológico & $80,1( \pm 15,5)$ & 83,3 & $89,1( \pm 30,2)$ & 83,3 & $84,4( \pm 11,3)$ & 87,5 & 0,390 \\
\cline { 2 - 7 } $\begin{array}{l}\text { Relações Sociais } \\
\text { Meio Ambiente }\end{array}$ & $69,8( \pm 11,4)$ & 68,7 & $80,6( \pm 22,0)$ & 75,0 & $67,8( \pm 10,0)$ & 68,7 & $<0,01$ \\
\hline
\end{tabular}

*Estimado pelo Teste de Kruska-Wallis; Med=mediana 
Tabela 4. Descrição dos domínios da qualidade de vida do WHOQOL-Old de acordo com a modalidade de atividade física praticada

\begin{tabular}{|c|c|c|c|c|c|c|c|}
\hline \multirow{2}{*}{$\begin{array}{c}\text { Domínios do WHO- } \\
\text { QOL-Old }\end{array}$} & \multicolumn{2}{|c|}{ Ginástica } & \multicolumn{2}{|c|}{ Hidroginástica } & \multicolumn{2}{|c|}{ Musculação } & \multirow{2}{*}{$p^{*}$} \\
\hline & Média (DP) & Med & Média (DP) & Med & Média (DP) & Med & \\
\hline $\begin{array}{l}\text { Funcionamento do } \\
\text { Sensório }\end{array}$ & $72,8( \pm 22,4)$ & 81,2 & $50,2( \pm 31,9)$ & 45,0 & $82,8( \pm 18,9)$ & 90,6 & $<0,01$ \\
\hline Autonomia & 66,5 & 7 & $76,3( \pm 19,6)$ & 75,0 & $11,7)$ & 9 & 100 \\
\hline $\begin{array}{l}\text { Atividades passadas, } \\
\text { presentes e futuras }\end{array}$ & $73, \varepsilon$ & 75,0 & 8 & 81,2 & $73,4( \pm 11,7)$ & 75,0 & 055 \\
\hline Participação Social & $75,6( \pm 16,1)$ & 75,0 & $75,4( \pm 17,7)$ & 75,0 & $71,5( \pm 15,1)$ & 68,5 & 0,426 \\
\hline Morte e Morrer & $62,9( \pm 24,5)$ & 68,7 & $40,9( \pm 37,2)$ & 31,2 & $76,6( \pm 25,0)$ & 81,2 & $<0,01$ \\
\hline Intimidade & $81,0( \pm 16,8)$ & 81,2 & $70,1( \pm 26,6)$ & 75,0 & $83,6( \pm 12,0)$ & 81,2 & 0,080 \\
\hline QV Total & $72,2( \pm 10,5)$ & 71,9 & $63,9( \pm 12,5)$ & 62,5 & $76,4( \pm 9,2)$ & 73,4 & $<0,01$ \\
\hline
\end{tabular}

*Estimado pelo Teste de Kruska-Wallis; Med=mediana

entre os praticantes de ginástica $(72,8 \pm 22,4$; Med $=81,2)$ e hidroginástica $(50,2 \pm 31,9$; Med $=45,0)$. No que diz respeito ao domínio morte e morrer, a maior média foi encontrada também entre os sujeitos praticantes de musculação $(76,6 \pm 25,0 ; M e d=81,2)$, seguido pela média dos praticantes de ginástica $(62,9 \pm 24,5$; Med $=68,7)$ e hidroginástica $(40,9 \pm 37,2$; Med=31,2). Com relação a avaliação geral da qualidade de vida, a maior média foi encontrada entre os praticantes de musculação $(76,4 \pm 9,2$; Med=73,4), seguidos pelos praticantes de ginástica $(72,2 \pm 10,5$; Med=71,9) e hidroginástica $(63,9 \pm 12,5$; Med=62,5).

No que tange as maiores médias encontradas entre os domínios de acordo com as modalidades, o domínio da intimidade, apresentou as maiores médias entre todos nas modalidades de ginástica $(81,0 \pm 16,8 ; \mathrm{Med}=81,2)$ e musculação $(83,6 \pm 12,0 ; \mathrm{Med}=81,2)$. Entre os praticantes de hidroginástica, o domínio com maior média foi o das atividades passadas, presentes e futuras $(85,3 \pm 26,1$; Med=81,2).
Considerando os domínios com as menores médias, o domínio morte e morrer foi o que apresentou as menores médias entre as modalidades de ginástica e hidroginástica. No entanto, entre os indivíduos que praticam musculação o domínio com menor média foi o da autonomia $(70,3 \pm 11,7 ;$ Med=71,9).

\section{DISCUSSÃO}

O presente estudo, que buscou verificar a influência da prática de atividade física nas modalidades ginástica, musculação e hidroginástica sobre a qualidade de vida de idosos, teve uma amostra predominantemente feminina e de quadro patológico, prevalecendo às doenças de ordem osteomusculares $(48,5 \%)$. Resultados similares foram descritos por Halaweh ${ }^{20}$ e coincidem como o descrito por Mazo et al. ${ }^{21}$ quando salientam que com o processo de envelhecimento, se observa um aumento da prevalência de doenças não transmissíveis. 
Apesar dos participantes do estudo apresentarem uma alta prevalência de enfermidades, os resultados indicaram que a maioria tem uma percepção positiva de sua saúde (boa ou ótima), o que pode estar relacionado com o tempo de participação nas atividades físicas em questão e vinculação com o programa. Segundo Corrêa et al. ${ }^{22}$, evidências epidemiológicas apontam que a atividade física é capaz de proporcionar uma série de modificações positivas na saúde física e mental dos praticantes. Choi et al. ${ }^{23}$ concordam com o comentado e citam, inclusive, que pode melhorar o quanto desfrutam da vida.

A visão positiva de saúde pode ser verificada refletida nos níveis de qualidade de vida totais observados $(67,4 \pm 12,6$ pontos), os quais coincidem com o estudo de Valadares et al. ${ }^{24}$ (66,9 $9 \pm 11,7$ pontos), em uma amostra de características semelhantes.

Se comparamos nossos resultados a aqueles descritos por Campos et al. ${ }^{25}$, que mediante a utilização da análise de Cluster em uma amostra de 2.052 idosos (59.7\% mulheres; média de 71 anos) consideraram valores aproximados a 65 pontos como fracos e 77 pontos como bons, verificamos resultados apenas regulares. Entretanto, se ponderamos que $76 \%$ de nossos participantes apresentavam dois ou mais quadros patológicos e que esta é uma variável que interfere diretamente na percepção de bem-estar ${ }^{25} \mathrm{e}$, ainda, que praticantes de atividade física revelam ter melhores valores de qualidade de vida, quando comparados a sedentários ${ }^{4,15,20}$, podemos considerar que nossos resultados são satisfatórios no que se refere à qualidade de vida.
Entre os domínios da qualidade de vida do WHOQOL-Bref, o que apresentou a maior média entre os demais foi o das relações sociais ( 86,4 pontos), assim como, em outros estudos ${ }^{26,27}$. Tal resultado poderia ser explicado a partir do tempo de participação no programa, uma vez que se estabelecem vínculos, o que repercutiu também na sua percepção positiva de sua saúde. Nos domínios do WHOQOL-Old, o que teve a maior média foi das atividades passadas, presentes e futuras (81,1 pontos), o que pode estar ligado ao desejo e a motivação que os participantes têm para enfrentar o que ainda está por vir. Talvez o fato de estar vinculado a um grupo ou ter uma percepção positiva de saúde, pode contribuir neste resultado.

Ao analisar nossos resultados por modalidade, observamos que a musculação apresenta resultados superiores frente às demais atividades analisadas, o que é compatível com o descrito na literatura quando estabelece a força muscular como uma das variáveis fundamentais na realização das atividades da vida diária e estabelece sua relação com o equilíbrio postural, indicador de independência em pessoas idosas ${ }^{4}$.

Ao considerarmos as médias de qualidade de vida de acordo com as modalidades, destacamos que os domínios das relações sociais e o das atividades passadas, presentes e futuras foram os que tiveram maiores médias entre todas. Entretanto, os domínios psicológico e morte e morrer foram aqueles que tiveram as piores médias.

Considerando o domínio das atividades passadas, presentes e futuras, ao des- 
crever a satisfação sobre conquistas na vida e os anseios individuais ${ }^{19}$, permite traçar um paralelo com um envelhecimento ativo, que independentemente do tipo de prática realizada, permite aos idosos uma percepção de bem estar mais positiva, e, por conseguinte, adquirem uma nova perspectiva de vida em um contexto voltado para um envelhecimento mais saudável.

Freitas et al. ${ }^{28}$ sinalizam que o elemento essencial relacionado ao envelhecimento bem-sucedido não se refere à preservação dos níveis de desempenho semelhantes a indivíduos mais jovens, mas a preservação do potencial para o desenvolvimento do indivíduo. Por outro lado, o domínio morte e morrer destacam às preocupações, inquietações e temores sobre a perspectiva de morrer e a maneira pela qual é enfrentada a proximidade desta fase da vida com o óbito ${ }^{19}$. Diferentemente de nossos achados, Carvalho et al. ${ }^{17}$ descrevem a autonomia como sendo seu resultado mais crítico (60 pontos), o que pode explicar-se pelo fato de seus participantes não apresentarem quadros patológicos associados.

Observando nossos resultados nos diferentes domínios, verificamos diferenças significativas no funcionamento do sensório $(p<0,01)$ e no morte e morrer $(p<0,01)$, salientando um comprometimento desses no grupo hidroginástica e na musculação, respectivamente. Fato que aparentemente nos surpreende, dado ser esta uma das modalidades de grande popularidade na população idosa ${ }^{25,28}$ e de maior tempo de permanência nas atividades (média de 9 anos). A inexistência de tantas diferenças significativas entre os grupos de cada uma das modalidades estudadas, nem referente a diferenças nas percepções de homens e mulheres e ausência de diferença significativa entre os grupos no referente a quadro patológico, hipotetizamos que pode existir uma relação entre o perfil psicológico, associado a fatores de medo, com a escolha da modalidade de prática. Esta hipótese se vê reforçada ao apresentar o menor resultado no funcionamento sensório (50,2 pontos), domínio diretamente relacionado à realização das atividades da vida diária. Das três modalidades, a hidroginástica é aquela que em nível de percepção, menor risco implica, seguida da ginástica e da "temida" musculação (modalidade relativamente recente em idosos $)^{29,30}$. Identificamos também diferença significativa no que diz respeito ao valor de qualidade de vida total, fornecido por meio do WHOQOL-Old $(p<0,01)$, assinalando valores superiores entre os participantes da musculação (83,6 pontos), quando comparados aos demais. Tal modalidade se distingue das demais, uma vez que apresentam menor idade, possuem menos comorbidades, fazem uso de menos medicamentos e tem uma percepção mais positiva de sua saúde, o que pode explicar tal resultado.

Nesse sentido, Tavares et al. ${ }^{26}$, salientam a importância de reinserir o idoso na sociedade, não apenas pelas atividades laborais, mas também em espaços que possibilitem a satisfação pessoal, fator que influencia na qualidade de vida dos idosos.

O domínio do meio ambiente esteve associado estatisticamente com as modali- 
dades $(p<0,01)$, sendo as maiores médias encontradas entre os indivíduos da hidroginástica, seguidas pela ginástica e musculação. Tal domínio tem a ver com o ambiente em que o indivíduo está inserido, em questão de infraestrutura disponível no dia a dia e destinada para as atividades de vida diária. Tal resultado diferiu de outros estudos ${ }^{26,27}$, que demonstraram que tal domínio apresentou os piores resultados, podendo estar relacionado com outros fatores não analisados aqui como a renda ou escolaridade dos sujeitos participantes.

\section{CONCLUSÕES}

Como principais resultados do presente estudo, destacamos uma amostra participante de um programa de atividade física universitário, predominantemente feminina, com idade até 70 anos, sem companheiro, com duas ou mais comorbidades, fazendo uso de dois medicamentos ou mais e com uma percepção positiva de saúde. Sobre a qualidade de vida, o domínio das relações sociais e das atividades passadas presentes e futuras foi o que apresentou as maiores médias da amostra. Os domínios psicológico e morte e morrer foram os que apresentaram as menores médias. Foi possível identificar que o tipo de modalidade em si, não influenciou diretamente na avaliação da qualidade de vida dos idosos, mas sim, os fatores pessoais sub- jetivos ou relacionados à condição de saúde dos sujeitos, como por exemplo, a idade mais avançada, o tempo de adesão ao programa, a presença de comorbidades e o elevado uso de medicamentos. Além disso, é importante ressaltar que a disparidade entre a quantidade de sujeitos em cada modalidade também é um fator a ser considerado nesta análise, o que pode ter influenciado no resultado.

Destacamos nesse sentido, que a modalidade de prática de atividade física é apenas uma variável a mais no universo subjetivo de avaliação da qualidade de vida e que, ainda, carecemos de outros estudos que nos auxiliem na compreensão de melhores estratégias de promoção de prática de atividade física atrelada à qualidade de vida.

De forma que seria interessante a realização de estudos que além de observar a qualidade de vida em diferentes modalidades, verificasse concomitantemente as capacidades físicas (força muscular e equilíbrio) e suas relações com as atividades de vida diária. Cabe destacar também, que este estudo apresentou limitações relativas ao tamanho da amostra e ao controle das intensidades utilizadas em cada uma das modalidades analisadas. Em contrapartida, entendemos que são poucos os estudos que contam com participantes com tanto tempo de prática orientada e com uma adesão de ao menos um $80 \%$ nas sessões de prática. 


\section{REFERÊNCIAS}

1. Milanovic Z, Pantelic S, Trajkovic, N, Sporis, G, Kostic R James, N. Age-related decrease in physical activity and functional fitness among elderly men and women. Clin Interv Aging. 2013; 8:549-556.

2. Zaitune MPA, Barros MBS, César CLG, Crandina L, Goldbaum M, Alves MCG. Fatores associados à prática de atividade física global e de lazer em idosos: Inquérito de Saúde no Estado de São Paulo (ISA-SP), Brasil. Cad Saude Publica. 2010; 26:1606-1618.

3. Farinatti PTV. Envelhecimento, promoção da saúde e exercício: bases teóricas e metodológicas, $1^{\text {a }}$ ed., Barueri, SP: Manole, 2008.

4. Svantesson U, Jones, J, Wolbert K, Alricsson M. Impact of Physical Activity on the Self-Perceived Quality of Life in Non-Frail Older Adults. J Clin Med Res. 2015; 7:585-593.

5. Antunes HK, Stella SG, Santos RF, Bueno OFA, De Mello MT. Depression, anxiety and quality of life scores in seniors after an endurance exercise program. Rev Bras Psiquiatr. 2015; 27:266-271.

6. Hupin D, Roche F, Gremeaux V et al. Even a low-dose of moderate-to-vigorous physical activity reduces mortality by $22 \%$ in adults aged $\geq 60$ years: a systematic review and meta-analysis. Br J Sports Med. 2015; 49: 1262-1267.

7. Mazo GZ. Atividade física, qualidade de vida e envelhecimento, $1^{\mathrm{a}}$ ed., Porto Alegre, RS: Sulina, 2008.

8. Madeira MC, Siqueira FCV, Facchini LA et al. Atividade física no deslocamento em adultos e idosos do Brasil: prevalências e fatores associados. Cad Saúde Publica. 2013; 29:165-174.

9. Nelson ME, Rejeski WJ; Blair SN et al. Physical activity and public health in older adults: Recommendation from the American College of Sports Medicine and the American Heart Association. Med Sci Sports Exerc. 2007; 39:1435-1445

10. Phoenix C, Grant B. Expanding the agenda for research on the physically active aging body. J Aging Phys Act. 2009; 17:362-79.

11. Carvalho ED, Valadares, ALR, Costa-Paiva LH, Pedro AO, Morais SS, Pinto-Neto AM. Atividade física e qualidade de vida em mulheres com 60 anos ou mais: fatores associados. Rev Bras Ginecol Obstet. 2010; 32:433-40.

12. Santos CAS, Dantas EEM, Moreira MHR. Correlation of physical aptitude, functional capacity, corporal balance and quality of life (QoL) among elderly women submitted to a post-menopausal physical activities program. Arch Gerontol Geriatr. 2011; 53:344-349.

13. Valadares ALR, Carvalho ED, Costa-Paiva LH, Morais SS, Pinto-Neto AM. Association between different types of physical activities and quality of life in women aged 60 years or over. Rev Assoc Med Bras. 2011; 57:450-455.
14. Nahas MV. Atividade física, saúde e qualidade de vida: conceitos e sugestões para um estilo de vida ativo, $4^{a}$ ed. Londrina, PR: Midiograf, 2006.

15. Sewo Sampaio PY, Sampaio RA, Coelho Júnior HJ et al. Differences in lifestyle, physical performance and quality of life between frail and robust Brazilian community-dwelling elderly women. Geriatr Gerontol Int. 2016 Jul;16(7):829-35

16. Neto GA, Pereira-Junior PP, Mura $G$ et al. Effects of different types of physical exercise on the perceived quality of life in active elderly. CNS Neurol Disord Drug Targets. 2015; 14:1152-1156.

17. Fransen HP, May AM, Beulens JW et al. Association between lifestyle factors and quality-adjusted life years in the EPIC-NL cohort. PLoS One. 2014; 9:e111480.

18. Fleck MPA, Louzada S, Xavier M, Chachamovich E, Vieira G, Santos L, et al. Aplicação da versão em português do instrumento abreviado de avaliação da qualidade de vida "WHOQOL-bref". Rev Saude Publica. 2000;34(2):178183.

19. Fleck MPA, Chachamovich E, Trentini, C. Development and validation of the Portuguese version of the $\mathrm{WHO}$ QOL-OLD module. Rev Saude Publica. 2006; 40:785-91.

20. Halaweh H, Willen C, Grimby-Ekman A, Svantesson U. Physical activity and health-related quality of life among community dwelling elderly. J Clin Med Res. 2015; 7:845852.

21. Mazo GZ, Lopes M, Benedetti TRB. Atividade Física e o Idoso: Concepção Gerontológica, $3^{\mathrm{a}}$ ed., Porto Alegre, RS: Sulina, 2009.

22. Corrêa LQ, Rombaldi AJ, Silva MC. Atividade Física e Sintomas do Envelhecimento Masculino em uma População do Sul do Brasil. Rev Bras Med Esporte. 2011; $17: 228-231$.

23. Choi M, Prieto-Merino D, Dale C et al. Effect of changes in moderate or vigorous physical activity on changes in health-related quality of life of elderly British women over seven years. Qual Life Res. 2013; 22:2011-2020.

24. Valadares ALR, Carvalho ED, Costa-Paiva LH, Morais SS, Pinto-Neto AM. Association between different types of physical activities and quality of life in women aged 60 years or over. Rev Assoc Med Bras. 2011; 57:450-455.

25. Campos AC, Ferreira e Ferreira E, Vargas AM, Albala C. Aging, gender and quality of life (AGEQOL) study: factors associated with good quality of life in older Brazilian community-dwelling adults. Health Qual Life Outcomes. 2014; 12:166-177.

26. Tavares DMS, Matias TGC, Ferreiras PCS, Pegorari MS Nascimento JS, Paiva MM. Qualidade de vida e autoestima de idosos na comunidade. Ciênc. Saúde Colet. 2016; 21(11):3557-3564. 
27. Ribeiro CG, Ferreti F, Sá CA. Qualidade de vida em função do nível de atividade física em idosos urbanos e rurais. Rev. Bras. Geriatr. Gerontol. 2017; 20(3):330-339.

28. Freitas CM, Santiago MS, Viana AT, Leão AC, Freyre C. Aspectos motivacionais que influenciam a adesão e manutenção de idosos a programas de exercícios físicos. Rev Bras Cineantropom Desempenho Hum 2007; 9:92100.

29. Takeshima N, Rogers ME, Watanabe E et al. Water-based exercise improves health-related aspects of fitness in older women. Med Sci Sports Exerc. 2002; 34:544-551.
30. Chase N, Xuemei S, Blair S. Comparison of the Health Aspects of Swimming With Other Types of Physical Activity and Sedentary Lifestyle Habits. JARE 2008; 2:151-161.

\section{CORRESPONDÊNCIA}

José Antonio Bicca Ribeiro

Mestre em Educação Física.

Doutorando do Programa de Pós-Graduação

em Educação Física

da Universidade Federal de Pelotas, Pelotas-RS, Brasil

Email: jantonio.bicca@gmail.com 